\title{
Two-photon absorption and resonant non-phase-matched second-harmonic generation in $\mathrm{CdSe}$
}

\author{
A. PENZKOFER, M. SCHÄFFNER, X. BAO \\ Naturwissenschaftliche Fakultät II - Physik, Universität Regensburg, \\ $D$-8400 Regensburg, FRG
}

Received 23 October 1989; accepted 15 February 1990

Exciting a hexagonal CdSe crystal with picosecond $\mathrm{Nd}$ : glass laser pulses, two-photon absorption and resonant non-phase-matched second-harmonic generation occur simultaneously. Using different crystal orientations, all components of the secondharmonic susceptibility tensor (non-vanishing components are $d_{31}, d_{33}$ and $d_{15}$ ) and some components of the two-photon absorption susceptibility tensor $\chi_{i j k l}^{(3) \prime}\left(-\omega_{L}\right.$; $\left.\omega_{L}, \omega_{L},-\omega_{L}\right)$ are determined.

\section{Introduction}

$\mathrm{CdSe}$ is a hexagonal crystal (wurtzite structure) of $\mathrm{P} 6_{3} \mathrm{mc}\left(\mathrm{C}_{6 \mathrm{v}}^{4}\right)$ space symmetry and $6 \mathrm{~mm}$ point symmetry [1]. Two-photon absorption (TPA) and second-harmonic generation (SHG) occur simultaneously when picosecond light pulses of a $\mathrm{Nd}$ : glass laser (wavelength $\left.\lambda_{\mathrm{L}}=1.054 \mu \mathrm{m}\right)$ are passed through the crystal.

$\mathrm{CdSe}$ has been applied previously to phase-matched SHG of $\mathrm{CO}_{2}$-laser light $\left(\lambda_{\mathrm{L}}=\right.$ $10.6 \mu \mathrm{m})$ [2] and the non-resonant second-order susceptibility components $d_{31}, d_{33}$ and $d_{15}$ have been determined. Phase-matched difference frequency mixing of $1.833 \mu \mathrm{m}(\mathrm{Nd}: \mathrm{YAG}$ laser) and $10.6 \mu \mathrm{m}\left(\mathrm{CO}_{2}\right.$-laser $)$ radiation was applied to generate light at $2.2 \mu \mathrm{m}$ [3]. The second-order susceptibility component $d_{33}$ has been determined under non-resonant $\left(\lambda_{\mathrm{L}}=2.12 \mu \mathrm{m}\right)$ [4] and resonant $\left(\lambda_{\mathrm{L}}=1.06 \mu \mathrm{m}\right)$ [5] conditions by non-phase-matched SHG. The reported second-harmonic susceptibility values are listed in Table I.

The TPA of neodymium laser pulses $\left(\lambda_{\mathrm{L}} \approx 1.06 \mu \mathrm{m}\right)$ in CdSe has been investigated in several papers [6-18]. The extracted TPA cross-sections are listed in Table II. Early measurements with long pump pulses gave rather large TPA cross-sections. The TPA depends on the crystal orientation and the light polarization (see below). The thirdorder non-linear susceptibility tensor $\chi_{i j k l}^{(3) \prime \prime}\left(-\omega_{\mathrm{L}} ; \omega_{\mathrm{L}}, \omega_{\mathrm{L}},-\omega_{\mathrm{L}}\right)$ is responsible for TPA $[19,20]$.

The contribution of resonant non-phase-matched SHG to the non-linear transmission of neodymium laser light through $\mathrm{CdSe}$ has been considered in $[10,11]$. The loss of pump light by the generation of second-harmonic light, was found to be approximately the same as the loss of pump light by TPA in the case of $\boldsymbol{E}_{\mathrm{L}} \| \boldsymbol{c}$ and $\boldsymbol{k}_{\mathrm{L}} \perp \boldsymbol{c}\left(\boldsymbol{E}_{\mathrm{L}}\right.$ is the electrical field strength of the pump laser, $\boldsymbol{c}$ is the optical axis of the crystal and $\boldsymbol{k}_{\mathrm{L}}$ is the wavevector of the pump laser). 
TABLE I Non-zero second-harmonic susceptibility components

\begin{tabular}{lllll}
\hline $\begin{array}{l}\lambda_{\mathrm{L}} \\
(\mu \mathrm{m})\end{array}$ & $\begin{array}{l}d_{15} \\
\left(\mathrm{mV}^{-1}\right)\end{array}$ & $\begin{array}{l}d_{31} \\
\left(\mathrm{mV}^{-1}\right)\end{array}$ & $\begin{array}{l}d_{33} \\
\left(\mathrm{mV}^{-1}\right)\end{array}$ & References \\
\hline 10.6 & $(3.1 \pm 0.75) \times 10^{-11}$ & $(2.85 \pm 0.63) \times 10^{-11}$ & $\begin{array}{l}(5.45 \pm 1.25) \times 10^{-11} \\
(7.5 \pm 0.9) \times 10^{-11}\end{array}$ & $\begin{array}{l}2 \\
5,22\end{array}$ \\
2.12 & & & $7.6 \times 10^{-11}$ & 5,22 \\
1.06 & & & $(3.9 \pm 0.3) \times 10^{-11}$ & This work \\
1.054 & $(2.3 \pm 0.3) \times 10^{-11}$ & $(2.5 \pm 0.3) \times 10^{-11}$ & & \\
\hline
\end{tabular}

The relationship between esu and SI values is $d(e s u)=\left(3 \times 10^{4} / 4 \pi\right) d\left(\mathrm{mV}^{-1}\right)$ [38].

TABLE II Two-photon absorption cross-sections of CdSe at $1.06 \mu \mathrm{m}$. Values were obtained by transmission measurements at room temperature

\begin{tabular}{|c|c|c|c|c|}
\hline $\begin{array}{l}\searrow\left(\boldsymbol{k}_{\mathrm{L}}, \boldsymbol{c}\right) \\
\text { (degrees) }\end{array}$ & $\begin{array}{l}\underset{\not}{\not}\left(\boldsymbol{E}_{\mathrm{L}}, \boldsymbol{c}\right) \\
\text { (degrees) }\end{array}$ & SHG & $\alpha^{(2)}\left(\mathrm{cm} \mathrm{W}^{-1}\right)$ & References \\
\hline Unknown & Unpolarized & noc & $(9.5 \pm 1) \times 10^{-7}$ & 6 \\
\hline Unknown & 90 & noc & $\alpha_{x x x x}^{(2)}=(3.9 \pm 0.4) \times 10^{-7}$ & 7 \\
\hline 90 & 0 & noc & $\alpha_{z z z z}^{(2)}=(9 \pm 0.9) \times 10^{-7}$ & 7 \\
\hline Unknown & 90 & noc & $\alpha_{x x x x}^{(2)}=2.2 \times 10^{-7}$ & 8,9 \\
\hline 90 & 0 & noc & $\alpha^{(2)}=2.1 \times 10^{-7}$ & 8,9 \\
\hline 0 & 90 & noo & $\alpha_{x x x x}^{(2)}=1.4 \times 10^{-7}$ & 10 \\
\hline 0 & 90 & noo & $\alpha_{x x x x}^{(2)}=1.3 \times 10^{-7}$ & $10^{*}$ \\
\hline 90 & 90 & con & $\alpha_{x x x x}^{(2)}=1.4 \times 10^{-7}$ & 10 \\
\hline 90 & 90 & con & $\alpha_{x x x x}^{(2)}=1.3 \times 10^{-7}$ & $10^{*}$ \\
\hline 90 & 0 & con & $\alpha_{z z z z}^{(2)}=6 \times 10^{-8}$ & 10 \\
\hline 90 & 0 & con & $\alpha_{z z z z}^{(2)} \approx 6 \times 10^{-8}$ & $10^{*}$ \\
\hline 0 & 90 & noo & $\alpha_{x x x x}^{(2)}=1.6 \times 10^{-7}$ & 11 \\
\hline 90 & 90 & con & $\alpha_{x x x x}^{(2)}=1.6 \times 10^{-7}$ & 11 \\
\hline 90 & 0 & con & $\alpha_{z z z z}^{(2)}=8 \times 10^{-8}$ & 11 \\
\hline Unknown & 90 & noc & $\alpha_{x x x x}^{(2)} \leqslant 2 \times 10^{-8}$ & 12 \\
\hline 90 & 0 & noc & $\alpha_{z z z z}^{(2)} \leqslant 2 \times 10^{-8}$ & 12 \\
\hline 0 & 90 & noo & $\alpha_{x x x x}^{(2)}=(3 \pm 0.5) \times 10^{-8}$ & 13 \\
\hline 0 & 90 & noo & $\alpha_{x x x x}^{(2)}=(4.8 \pm 1.4) \times 10^{-8}$ & 14 to 16 \\
\hline 0 & 90 & noo & $\alpha_{x x x x}^{(2)}=(1.8 \pm 0.3) \times 10^{-8}$ & 17 \\
\hline 0 & 90 & noo & $\alpha_{x x x x}^{(2)}=(1.8 \pm 0.3) \times 10^{-8}$ & This work \\
\hline 90 & 90 & con & $\alpha_{x x x x}^{(2)}=(1.8 \pm 0.3) \times 10^{-8}$ & This work \\
\hline 90 & 0 & con & $\alpha_{z z z z}^{(2)}=(1.8 \pm 0.3) \times 10^{-8}$ & This work \\
\hline \multirow[t]{2}{*}{90} & 45 & con & $\alpha_{x z z x}^{(2)}+\alpha_{x z x z}^{(2)}=(3 \pm 3) \times 10^{-9}$ & This work \\
\hline & & & $\alpha_{z x x z}^{(2)}+\alpha_{z x z x}^{(2)}=(3 \pm 3) \times 10^{-9}$ & This work \\
\hline
\end{tabular}

*Temperature is $80 \mathrm{~K}$. SHG, second-harmonic generation; noc, not considered; noo, no occurrence; con, considered.

An appreciable influence of excited-state absorption on the non-linear transmission was reported in [11, 12]. Q-switched lasers with pulse durations of 15 [11] and $20 \mathrm{~ns}$ [12] were used. Approximately $20 \%$ of the absorption was found to be due to excited-state absorption of the two-photon-generated carriers [11].

In this paper the TPA and the resonant non-phase-matched SHG in CdSe are studied. Single picosecond light pulses of a passively mode-locked $\mathrm{Nd}$-phosphate glass laser are applied (wavelength $\lambda_{\mathrm{L}}=1.054 \mu \mathrm{m}$, pulse duration $\Delta t_{\mathrm{L}} \approx 6 \mathrm{ps}$ ). Using different crystal orientations, all non-zero components of the second-order non-linear susceptibility tensor responsible for $\mathrm{SHG}$ are determined. For the TPA only some components of the third-order non-linear susceptibility tensor $\chi_{i j k l}^{(3) \prime}\left(-\omega_{\mathrm{L}} ; \omega_{\mathrm{L}}, \omega_{\mathrm{L}},-\omega_{\mathrm{L}}\right)$ are determined. Our measurements indicate no measurable contribution of excited-state absorption to the non-linear 
transmission. The SHG is found to be too weak to contribute measurably to the non-linear pump-pulse absorption.

\section{Theory}

The electrical field strengths $\boldsymbol{E}_{\mathrm{L}}=\frac{1}{2}\left\{\boldsymbol{E}_{\mathrm{L} 0} \exp \left[\mathrm{i}\left(\omega_{\mathrm{L}} t-k_{\mathrm{L}} s\right)\right]+\right.$ c.c. $\}$ of the pump laser and $\boldsymbol{E}_{2}=\frac{1}{2}\left\{\boldsymbol{E}_{20} \exp \left[\mathrm{i}\left(\omega_{2} t-k_{2} s\right)\right]+\right.$ c.c. $\}$ of the second-harmonic light are considered. $s$ is the propagation direction of the laser light. The discussion will be restricted to light propagation parallel to the optical axis $(\boldsymbol{k} \| \boldsymbol{c}, s=z)$ and perpendicular to the optical axis $(\boldsymbol{k} \perp \boldsymbol{c}$, $s=y, x$ and $y$ are equivalent) of the CdSe crystal. The resonant ( $\omega_{2}$ in the absorption region) non-phase-matched $\left(k_{2} \neq 2 k_{\mathrm{L}}\right)$ collinear SHG and the TPA are included in the analysis. The excited-state absorption of photogenerated carriers is not included in the theory, since the experiments give no indication of excited-state absorption under our experimental conditions (for the inclusion of excited-state absorption, see [21]). In the slowly varying amplitude approximation the wave equations for $\boldsymbol{E}_{20}$ and $\boldsymbol{E}_{\mathrm{L} 0}$ are [21-23]

$$
\begin{aligned}
\frac{\partial}{\partial s^{\prime}} E_{20, i}+\frac{\alpha_{2, i}}{2} E_{20, i} & =-\mathrm{i} \frac{\omega_{2} \mu_{0} c_{0}}{2 n_{2, i}} P_{20, i}^{(2)} \exp \left(\mathrm{i} \Delta k_{i} s^{\prime}\right) \\
\frac{\partial}{\partial s^{\prime}} E_{\mathrm{L} 0, i}+\frac{\alpha_{\mathrm{L}, i}}{2} E_{\mathrm{L} 0, i} & =-\mathrm{i} \frac{\omega_{\mathrm{L}} \mu_{0} c_{0}}{2 n_{\mathrm{L}, i}}\left[P_{\mathrm{L} 0, i}^{(2)} \exp \left(-\mathrm{i} \Delta k_{i} s^{\prime}\right)+P_{\mathrm{L} 0, i}^{(3)}\right]
\end{aligned}
$$

The index $i$ denotes $x, y$ or $z ; \mu_{0}$ is the permeability of free space; $c_{0}$ is the speed of light in vacuo; $\omega_{\mathrm{L}}=2 \pi v_{\mathrm{L}}=2 \pi c_{0} / \lambda_{\mathrm{L}}=2 \pi c_{0} \tilde{v}_{\mathrm{L}}$ is the angular frequency of the pump light. $\omega_{2}=2 \omega_{\mathrm{L}}$ is the angular frequency of the second-harmonic light. $n_{\mathrm{L}, i}$ and $n_{2, i}$ are the refractive indices at the fundamental and second-harmonic frequency, respectively. The moving-frame transformations $t_{\mathrm{L}}^{\prime}=t-n_{\mathrm{L}, i} s / c_{0}, t_{2}^{\prime}=t-n_{2, i} s / c_{0}$ and $s^{\prime}=s$ have been used. $t$ is the time.

The second-order non-linear polarization $\boldsymbol{P}_{2}^{(2)}=\frac{1}{2}\left\{\boldsymbol{P}_{20}^{(2)} \exp \left[\mathrm{i}\left(\omega_{2} t-k_{2}^{\mathrm{P}} s\right)\right]+\right.$ c.c. $\}$ responsible for SHG (Equation 1) is given by

$$
P_{20, i}^{(2)}=\varepsilon_{0} \sum_{j, k} \chi_{i j k}^{(2)}\left(-\omega_{2} ; \omega_{\mathrm{L}}, \omega_{\mathrm{L}}\right) E_{\mathrm{L} 0, j} E_{\mathrm{L} 0, k}
$$

where $\varepsilon_{0}$ is the permittivity of free space. The polarization wavevector is $k_{2}^{\mathrm{P}}=k_{\mathrm{L}, j}+k_{\mathrm{L}, k}$. The wavevector mismatch $\Delta k_{i}$ (Equations 1 and 2) is

$$
\Delta k_{i}=k_{2, i}-k_{2}^{\mathrm{P}}=k_{2, i}-k_{\mathrm{L}, j}-k_{\mathrm{L}, k}=\omega_{\mathrm{L}}\left(2 n_{2, i}-n_{\mathrm{L}, j}-n_{\mathrm{L}, k}\right) / c_{0}
$$

The second-harmonic susceptibility tensor $\chi^{(2)}\left(-\omega_{2} ; \omega_{\mathrm{L}}, \omega_{\mathrm{L}}\right)$ of $\mathrm{CdSe}(6 \mathrm{~mm}$ point group) has three different non-zero components $[22,24]$ which are given by $\chi_{x x z}^{(2)}=\chi_{x=x}^{(2)}=\chi_{y y z}^{(2)}=$ $\chi_{y z y}^{(2)}=d_{15}, \chi_{z x x}^{(2)}=\chi_{z y y}^{(2)}=d_{31}$ and $\chi_{z z z}^{(2)}=d_{33}$. All other components are zero. In contracted matrix form $\chi^{(2)}\left(-\omega_{2} ; \omega_{\mathrm{L}}, \omega_{\mathrm{L}}\right)$ is

$$
\begin{aligned}
& \chi^{(2)}\left(-\omega_{2} ; \omega_{\mathrm{L}}, \omega_{\mathrm{L}}\right)=\left(\begin{array}{cccccc}
1 & 2 & 3 & 4 & 5 & 6 \\
\chi_{x x x} & \chi_{x y y} & \chi_{x z z} & \chi_{x y z} & \chi_{x x z} & \chi_{x x y} \\
\chi_{y x x} & \chi_{y y y} & \chi_{y z z} & \chi_{y y z} & \chi_{y x z} & \chi_{y: x y} \\
\chi_{z x x} & \chi_{z y y} & \chi_{z z z} & \chi_{z y z} & \chi_{z x x} & \chi_{z x y}
\end{array}\right) \begin{array}{l}
1 \\
2 \\
3
\end{array} \\
& =\left(\begin{array}{cccccc}
0 & 0 & 0 & 0 & d_{15} & 0 \\
0 & 0 & 0 & d_{15} & 0 & 0 \\
d_{31} & d_{31} & d_{33} & 0 & 0 & 0
\end{array}\right)
\end{aligned}
$$


The second-order non-linear polarization $\boldsymbol{P}_{\mathrm{L}}^{(2)}=\frac{1}{2}\left\{\boldsymbol{P}_{\mathrm{L} 0}^{(2)} \exp \left[\mathrm{i}\left(\omega_{\mathrm{L}} t-k_{\mathrm{L}}^{\mathrm{P}} s\right)\right]+\right.$ c.c. $\}$ of Equation 2 is given by

$$
P_{\mathrm{L} 0, i}^{(2)}=2 \varepsilon_{0} \sum_{j, k} \chi_{i j k}^{(2)}\left(-\omega_{\mathrm{L}} ; \omega_{2},-\omega_{\mathrm{L}}\right) E_{20, j} E_{\mathrm{L} 0, k}^{*}
$$

with [24]

$$
\chi_{i j k}^{(2)}\left(-\omega_{\mathrm{L}} ; \omega_{2},-\omega_{\mathrm{L}}\right)=\chi_{j i k}^{(2) *}\left(-\omega_{2} ; \omega_{\mathrm{L}}, \omega_{\mathrm{L}}\right)
$$

and

$$
k_{\mathrm{L}}^{\mathrm{P}}=k_{2 j}-k_{\mathrm{L}, k}
$$

The third-order non-linear polarization $\boldsymbol{P}_{\mathrm{L}}^{(3)}=\frac{1}{2}\left\{\boldsymbol{P}_{\mathrm{L} 0}^{(3)} \exp \left[\mathrm{i}\left(\omega_{\mathrm{L}} t-k_{\mathrm{L}} s\right)\right]+\right.$ c.c. $\}$ responsible for TPA (Equation 2) is

$$
P_{\mathrm{L} 0, i}^{(3)}=-\mathrm{i} 3 \varepsilon_{0} \sum_{j, k, l} \chi_{i j k l}^{(3) \prime \prime}\left(-\omega_{\mathrm{L}} ; \omega_{\mathrm{L}}, \omega_{\mathrm{L}},-\omega_{\mathrm{L}}\right) E_{\mathrm{L} 0, j} E_{\mathrm{L} 0, k} E_{\mathrm{L} 0, l}^{*}
$$

Only the imaginary part $\chi^{(3) \prime \prime}$ of $\chi^{(3)}=\chi^{(3) \prime}-\mathrm{i} \chi^{(3) \prime \prime}$ contributes to TPA. The real part $\chi^{(3) \prime}$ causes phase changes that are not considered here. The non-vanishing third-order susceptibility components of CdSe (6 mm point group) are [24]: $\chi_{z z z z}, \chi_{x x x x}=\chi_{y y y y}=\chi_{x x y y}+$ $\chi_{x y y x}+\chi_{x y x y}, \chi_{y y x x}=\chi_{x x y y}, \chi_{y x x y}=\chi_{x y y x}, \chi_{y x y x}=\chi_{x y x y}, \chi_{y y z z}=\chi_{x x z z}, \chi_{z z y y}=\chi_{z z x x}, \chi_{z y y z}=$ $\chi_{z x x z}, \chi_{y z z y}=\chi_{x z z x}, \chi_{y z y z}=\chi_{x z x z}$ and $\chi_{z y z y}=\chi_{z x z x}$.

Four different experimental arrangements are studied: (1) $\boldsymbol{k}_{\mathrm{L}} \| c$-axis, (2) $\boldsymbol{k}_{\mathrm{L}} \perp c$-axis and $\boldsymbol{E}_{\mathrm{L}} \perp c$-axis, (3) $\boldsymbol{k}_{\mathrm{L}} \perp c$-axis and $\boldsymbol{E}_{\mathrm{L}} \| c$-axis, and (4) $\boldsymbol{k}_{\mathrm{L}} \perp c$-axis and $\measuredangle\left(\boldsymbol{E}_{\mathrm{L}}, c\right.$-axis $)=45^{\circ}$. The differential equation systems for these arrangements are given in the following.

\section{1. $\boldsymbol{k}_{\mathrm{L}}\left\|\boldsymbol{z}, E_{\mathrm{L}}\right\| \boldsymbol{x}$ (equivalent to $E_{\mathrm{L}} \| \boldsymbol{y}$ )}

The fundamental field strength is $\boldsymbol{E}_{\mathrm{L}}=\left(E_{\mathrm{L}}, 0,0\right)$. No SHG occurs since $\chi_{x x x}^{(2)}=\chi_{y x x}^{(2)}=0$. Equations 1 to 7 reduce to

$$
\frac{\partial}{\partial z} E_{\mathrm{L} 0}+\frac{\alpha_{\mathrm{L} o}}{2} E_{\mathrm{L} 0}=-\frac{3 \omega_{\mathrm{L}}}{2 n_{\mathrm{Lo}} c_{0}} \chi_{x x x x}^{(3) \prime \prime} E_{\mathrm{L} 0}^{2} E_{\mathrm{L} 0}^{*}
$$

$\alpha_{\mathrm{Lo}}$ is the linear ordinary absorption coefficient and $n_{\mathrm{Lo}}$ is the ordinary refractive index at the laser frequency $\omega_{\mathrm{L}}$.

With the intensity relation $I=n \varepsilon_{0} c_{0}\left|E_{0}\right|^{2} / 2$, Equation 8 may be transformed to

$$
\frac{\partial}{\partial z} I_{\mathrm{L}}+\alpha_{\mathrm{Lo}} I_{\mathrm{L}}=-\alpha_{x x x x}^{(2)} I_{\mathrm{L}}^{2}
$$

where the ordinary TPA coefficient $\alpha_{x x x x}^{(2)}$ is given by

$$
\alpha_{x x x x x}^{(2)}=\frac{6 \omega_{\mathrm{L}}}{n_{\mathrm{Lo}}^{2} c_{0}^{2} \varepsilon_{0}} \chi_{x x x x x}^{(3) \prime \prime}\left(-\omega_{\mathrm{L}} ; \omega_{\mathrm{L}}, \omega_{\mathrm{L}},-\omega_{\mathrm{L}}\right)
$$

The solution of Equation 9 is

$$
I_{\mathrm{L}}(z)=\frac{I_{\mathrm{L}}(0) \exp \left(-\alpha_{\mathrm{Lo}} z\right)}{1+\alpha_{x x x x}^{(2)} I_{\mathrm{L}}(0)\left[1-\exp \left(-\alpha_{\mathrm{Lo}} z\right)\right] / \alpha_{\mathrm{Lo}}}
$$


The intensity transmission, $T_{1}=I_{\mathrm{L}, \text { out }} / I_{\mathrm{L}, \text { in }}=I_{\mathrm{L}}(l)\left(1-R_{\mathrm{Lo}}\right) /\left[I_{\mathrm{L}}(0) /\left(1-R_{\mathrm{Lo}}\right)\right]$, is

$$
T_{\mathrm{I}}=\frac{\left(1-R_{\mathrm{Lo}}\right)^{2}}{1-R_{\mathrm{Lo}}^{2} \exp \left(-2 \alpha_{\mathrm{Lo}} l\right)} \frac{\exp \left(-\alpha_{\mathrm{Lo}} l\right)}{1+\alpha_{x x x x}^{(2)}\left(1-R_{\mathrm{Lo}}\right) I_{\mathrm{L}, \text { in }}\left[1-\exp \left(-\alpha_{\mathrm{Lo}} l\right)\right] / \alpha_{\mathrm{Lo}}}
$$

$R_{\mathrm{Lo}}$ is the reflectivity of the ordinary polarized pump light. The first factor includes multiple reflections. Two-photon absorption is taken into account only for the first passage. The energy transmission, $T_{\mathrm{E}}=W_{\mathrm{L} \text {, out }} / W_{\mathrm{L}, \text { in }}$, is obtained by integrating over the temporal and spatial intensity distribution. For a Gaussian input pulse, $I_{\mathrm{L}, \text { in }}\left(r, t^{\prime}\right)=I_{0 \mathrm{~L}} \exp \left[-\left(r / r_{0}\right)^{2}-\right.$ $\left.\left(t^{\prime} \mid t_{0}\right)^{2}\right]$, the energy transmission is

$$
\begin{aligned}
T_{\mathrm{E}} & =\frac{\int_{0}^{\infty} r \mathrm{~d} r \exp \left[-\left(r / r_{0}\right)^{2}\right] \int_{-\infty}^{\infty} \mathrm{d} t^{\prime} \exp \left[-\left(t^{\prime} / t_{0}\right)^{2}\right] T_{1}\left(r, t^{\prime}\right)}{\int_{0}^{\infty} r \mathrm{~d} r \exp \left[-\left(r / r_{0}\right)^{2}\right] \int_{-\infty}^{\infty} \mathrm{d} t^{\prime} \exp \left[-\left(t^{\prime} \mid t_{0}\right)^{2}\right]} \\
& =4 \pi^{-1 / 2} \int_{0}^{\infty} r \mathrm{~d} r \exp \left(-r^{2}\right) \int_{0}^{\infty} \mathrm{d} t^{\prime} \exp \left(-t^{\prime 2}\right) T_{1}\left(r / r_{0}, t^{\prime} / t_{0}\right)
\end{aligned}
$$

In the experiments $T_{\mathrm{E}}$ is measured. $\alpha_{x x x x}^{(2)}$ is determined by fitting Equation 13 to experimental transmissions.

\section{2. $\boldsymbol{k}_{\mathrm{L}}\left\|\boldsymbol{y}, \boldsymbol{E}_{\mathrm{L}}\right\| \boldsymbol{x}$ (oo $\rightarrow$ e interaction)}

The fundamental field strength is $\boldsymbol{E}_{\mathrm{L}}=\left(E_{\mathrm{L}}, 0,0\right)$. Second-harmonic light is generated with $\boldsymbol{E}_{2}=\left(0,0, E_{2}\right)$. Equations 1 to 7 reduce to

$$
\begin{aligned}
\frac{\partial}{\partial y} E_{20}+\frac{\alpha_{2 \mathrm{e}}}{2} E_{20} & =-\mathrm{i} \frac{\omega_{2}}{2 n_{2 \mathrm{e}} c_{0}} d_{31} E_{\mathrm{L} 0}^{2} \exp \left(\mathrm{i} \Delta k_{\mathrm{ooe}} y\right) \\
\frac{\partial}{\partial y} E_{\mathrm{L} 0}+\frac{\alpha_{\mathrm{Lo}}}{2} E_{\mathrm{L} 0} & =-\mathrm{i} \frac{\omega_{\mathrm{L}}}{2 n_{\mathrm{Lo}} c_{0}}\left[2 d_{31}^{*} E_{20} E_{\mathrm{L} 0}^{*} \exp \left(-\mathrm{i} \Delta k_{\mathrm{ooe}} y\right)-\mathrm{i} 3 \chi_{x x x x x}^{(3) \prime \prime}\left|E_{\mathrm{L} 0}\right|^{2} E_{\mathrm{L} 0}\right] \\
& =-\mathrm{i} \frac{\omega_{\mathrm{L}}}{n_{\mathrm{Lo}} c_{0}} d_{31}^{*} E_{20} E_{\mathrm{L} 0}^{*} \exp \left(-\mathrm{i} \Delta k_{\mathrm{ooe}} y\right)-\frac{1}{2} \alpha_{x x x x}^{(2)} I_{\mathrm{L}} E_{\mathrm{L} 0}
\end{aligned}
$$

with $\Delta k_{\mathrm{ooe}}=2 \omega_{\mathrm{L}}\left(n_{2 \mathrm{e}}-n_{\mathrm{Lo}}\right) / c_{0}$.

Equations $14 \mathrm{a}$ and $\mathrm{b}$ have to be solved numerically in the general case of pump-pulse depletion by SHG and TPA.

For our experimental conditions Equations $14 \mathrm{a}$ and $\mathrm{b}$ may be simplified. It is found that the pump-pulse reduction by SHG is negligible. Therefore, Equation $14 \mathrm{~b}$ reduces to Equation 9, and the pump-pulse transmission is given by Equations 12 and 13. In the case of constant pump light $\left(E_{\mathrm{L} 0}(y)=\right.$ constant; that is, $\alpha_{\mathrm{Lo}}=0$ and $\left.\alpha_{x x x x}^{(2)}=0\right)$ the solution of Equation $14 \mathrm{a}$ is [23]

$$
E_{20}(y)=-\mathrm{i} \frac{\omega_{2} d_{31}}{2 n_{2 \mathrm{e}} c_{0}} E_{\mathrm{L} 0}^{2} \exp \left(-\frac{1}{2} \alpha_{2 \mathrm{e}} y\right) \frac{\exp \left[\left(\frac{1}{2} \alpha_{2 \mathrm{e}}+\mathrm{i} \Delta k_{\mathrm{ooe}}\right) y\right]-1}{\frac{1}{2} \alpha_{2 \mathrm{e}}+\mathrm{i} \Delta k_{\mathrm{ooe}}}
$$

and the second-harmonic intensity is

$$
I_{2}(y)=\frac{\omega_{2}^{2}\left|d_{31}\right|^{2} I_{\mathrm{L}}^{2}}{2 n_{2 \mathrm{e}} n_{\mathrm{Lo}}^{2} c_{0}^{3} \varepsilon_{0}} \frac{1+\exp \left(-\alpha_{2 \mathrm{e}} y\right)-2 \cos \left(\Delta k_{\text {ooe }} y\right) \exp \left(-\alpha_{2 \mathrm{e}} y / 2\right)}{\left(\alpha_{2 \mathrm{e}} / 2\right)^{2}+\Delta k_{\mathrm{ooe}}^{2}}
$$


For our situation the absorption coefficients $\alpha_{2 \mathrm{e}}\left(\boldsymbol{E}_{2} \| \boldsymbol{z}\right)$ and $\alpha_{2 \mathrm{o}}\left(\boldsymbol{E}_{2} \| \boldsymbol{x}\right)$ are very large [25] and the pump laser intensity is reduced by TPA and linear absorption. Because of these conditions the output second-harmonic intensity $I_{2 \text {, out }}=I_{2}(l)\left(1-R_{2 \mathrm{e}}\right)$ is obtained by replacing $I_{\mathrm{L}}$ by $I_{\mathrm{L}}(l)=I_{\mathrm{L}, \text { out }} /\left(1-R_{\mathrm{Lo}}\right) \cdot R_{2 \mathrm{e}}$ is the reflectivity of the extraordinary polarized second-harmonic light and $R_{\mathrm{Lo}}$ is the reflectivity of the ordinary polarized fundamental light. Equation 16 reduces to

$$
\begin{aligned}
I_{2, \text { out }}= & \left(1-R_{2 \mathrm{e}}\right) \frac{\omega_{2}^{2}\left|d_{31}\right|^{2} I_{\mathrm{L}}^{2}(l)}{2 n_{2 \mathrm{e}} n_{\mathrm{Lo}}^{2} c_{0}^{3} \varepsilon_{0}} \frac{1}{\left(\alpha_{2 \mathrm{e}} / 2\right)^{2}+\Delta k_{\mathrm{ooe}}^{2}} \\
= & \left(1-R_{2 \mathrm{e}}\right)\left(1-R_{\mathrm{Lo}}\right)^{2} \frac{\omega_{2}^{2}\left|d_{31}\right|^{2} I_{\mathrm{L}, \text { in }}^{2}}{2 n_{2 \mathrm{e}} n_{\mathrm{Lo}}^{2} c_{0}^{3} \varepsilon_{0}} \\
& \times \frac{\exp \left(-2 \alpha_{\mathrm{Lo}} l\right)}{\left\{1+\alpha_{x x x x}^{(2)}\left(1-R_{\mathrm{Lo}}\right) I_{\mathrm{L}, \text { in }}\left[1-\exp \left(-\alpha_{\mathrm{Lo}} l\right)\right] / \alpha_{\mathrm{Lo}}\right\}^{2}} \frac{1}{\left(\alpha_{2 \mathrm{e}} / 2\right)^{2}+\Delta k_{\mathrm{ooe}}^{2}}
\end{aligned}
$$

The second-harmonic energy conversion efficiency, $\eta_{\mathrm{E}}^{\mathrm{SH}}=W_{2, \text { out }} / W_{\mathrm{L}, \text { in }}$, is

$$
\eta_{\mathrm{E}}^{\mathrm{SH}}=\frac{\int_{0}^{\infty} r \mathrm{~d} r \int_{-\infty}^{\infty} \mathrm{d} t^{\prime} I_{2, \text { out }}\left(r, t^{\prime}\right)}{I_{0 \mathrm{~L}, \text { in }} \int_{0}^{\infty} r \mathrm{~d} r \exp \left(-r^{2} / r_{0}^{2}\right) \int_{-\infty}^{\infty} \mathrm{d} t^{\prime} \exp \left(-t^{\prime 2} / t_{0}^{2}\right)}
$$

The TPA coefficient $\alpha_{x x . x x}^{(2)}$ is determined by fitting Equation 13 to the experimental energy transmissions. The susceptibility component $\left|d_{31}\right|$ is obtained by fitting Equation 18 to experimental second-harmonic energy conversion efficiencies.

\section{3. $\boldsymbol{k}_{\mathrm{L}}\left\|\boldsymbol{y}, \boldsymbol{E}_{\mathrm{L}}\right\| \boldsymbol{z}$ (ee $\rightarrow$ e interaction)}

The fundamental field strength is $E_{\mathrm{L}}=\left(0,0, E_{\mathrm{L}}\right)$. The field strength of the generated second-harmonic light is $\boldsymbol{E}_{2}=\left(0,0, E_{2}\right)$. Equations 1 to 7 reduce to

$$
\begin{aligned}
\frac{\partial}{\partial y} E_{20}+\frac{\alpha_{2 \mathrm{e}}}{2} E_{20} & =-\mathrm{i} \frac{\omega_{2}}{2 n_{2 \mathrm{e}} c_{0}} d_{33} E_{\mathrm{L} 0}^{2} \exp \left(\mathrm{i} \Delta k_{\text {eee }} y\right) \\
\frac{\partial}{\partial y} E_{\mathrm{L} 0}+\frac{\alpha_{\mathrm{Le}}}{2} E_{\mathrm{L} 0} & =-\frac{\mathrm{i} \omega_{\mathrm{L}}}{2 n_{\mathrm{Le}} c_{0}}\left[2 d_{33}^{*} E_{20} E_{\mathrm{L} 0}^{*} \exp \left(-\mathrm{i} \Delta k_{\text {eee }} y\right)-\mathrm{i} 3 \chi_{z z z z}^{(3) \prime}\left|E_{\mathrm{L} 0}\right|^{2} E_{\mathrm{L} 0}\right] \\
& =-\frac{\mathrm{i} \omega_{\mathrm{L}}}{n_{\mathrm{Le}} c_{0}} d_{33}^{*} E_{20} E_{\mathrm{L} 0}^{*} \exp \left(-\mathrm{i} \Delta k_{\text {eee }} y\right)-\frac{1}{2} \alpha_{z z z z}^{(2)} I_{\mathrm{L}} E_{\mathrm{L} 0}
\end{aligned}
$$

with $\Delta k_{\text {eee }}=2 \omega_{\mathrm{L}}\left(n_{2 \mathrm{e}}-n_{\mathrm{Le}}\right) / c_{0}$ and

$$
\alpha_{z z z z}^{(2)}=\frac{6 \omega_{\mathrm{L}}}{n_{\mathrm{Le}}^{2} c_{0}^{2} \varepsilon_{0}} \chi_{z z z z}^{(3) \prime \prime}\left(-\omega_{\mathrm{L}} ; \omega_{\mathrm{L}}, \omega_{\mathrm{L}},-\omega_{\mathrm{L}}\right)
$$

For negligible pump-pulse depletion by SHG the transmitted pump-pulse intensity is (see Equation 11)

$$
I_{\mathrm{L}}(l)=\frac{I_{\mathrm{L}, \text { in }}\left(1-R_{\mathrm{Le}}\right) \exp \left(-\alpha_{\mathrm{Le}} l\right)}{1+\alpha_{z z z z}^{(2)} I_{\mathrm{L}, \text { in }}\left(1-R_{\mathrm{Le}}\right)\left[1-\exp \left(-\alpha_{\mathrm{Le}} l\right)\right] / \alpha_{\mathrm{Le}}}
$$


and the second-harmonic output intensity is (see Equation 17)

$$
\begin{aligned}
I_{2, \text { out }}= & \left(1-R_{2 \mathrm{e}}\right)\left(1-R_{\mathrm{Le}}\right)^{2} \frac{\omega_{2}^{2}\left|d_{33}\right|^{2} I_{\mathrm{L}, \text { in }}^{2}}{2 n_{2 \mathrm{e}} n_{\mathrm{Le}}^{2} c_{0}^{3} \varepsilon_{0}} \\
& \times \frac{\exp \left(-2 \alpha_{\mathrm{Le}} l\right)}{\left\{1+\alpha_{z z z z}^{(2)}\left(1-R_{\mathrm{Le}}\right) I_{\mathrm{L}, \text { in }}\left[1-\exp \left(-\alpha_{\mathrm{Le}} l\right)\right] / \alpha_{\mathrm{Le}}\right\}^{2}} \frac{1}{\left(\alpha_{2 \mathrm{e}} / 2\right)^{2}+\Delta k_{\mathrm{eee}}^{2}}
\end{aligned}
$$

\section{4. $k_{\mathrm{L}} \| \boldsymbol{y}, E_{\mathrm{L}}$ diagonal to $x$ - and $z$-axes (oe $\rightarrow$ o interaction)}

The input fundamental field strength is $E_{\mathrm{L}}=\left(E_{\mathrm{L}, x}, 0, E_{\mathrm{L}, z}\right)=2^{-1 / 2} E_{\mathrm{L}}(1,0,1)$. The field strength of the generated second harmonic light is $\boldsymbol{E}_{2}=\left(E_{2}, 0,0\right)$. Equations 1 to 7 reduce to

$$
\begin{aligned}
\frac{\partial}{\partial y} E_{20}+\frac{\alpha_{20}}{2} E_{20}= & -\mathrm{i} \frac{\omega_{2}}{n_{20} c_{0}} d_{15} E_{\mathrm{L} 0 x} E_{\mathrm{L} 0 z} \exp \left(\mathrm{i} \Delta k_{\text {oеo }} y\right) \\
\frac{\partial}{\partial y} E_{\mathrm{L} 0 x}+\frac{\alpha_{\mathrm{Lo}}}{2} E_{\mathrm{L} 0 x}= & -\mathrm{i} \frac{\omega_{\mathrm{L}}}{n_{\mathrm{Lo}} c_{0}} d_{15}^{*} E_{20} E_{\mathrm{L} 0 z}^{*} \exp \left(-\mathrm{i} \Delta k_{\mathrm{oeo}} y\right) \\
& -\frac{1}{2} E_{\mathrm{L} 0 x}\left[\alpha_{x x x x}^{(2)} I_{\mathrm{L} x}+\left(\alpha_{x z z x}^{(2)}+\alpha_{x z x z}^{(2)}\right) I_{\mathrm{L} z}\right] \\
\frac{\partial}{\partial y} E_{\mathrm{L} 0 z}+\frac{\alpha_{\mathrm{Le}}}{2} E_{\mathrm{L} 0 z}= & -\mathrm{i} \frac{\omega_{\mathrm{L}}}{n_{\mathrm{Le}} c_{0}} d_{15}^{*} E_{20} E_{\mathrm{L} 0 x}^{*} \exp \left(-\mathrm{i} \Delta k_{\mathrm{oeo}} y\right) \\
& -\frac{1}{2} E_{\mathrm{L} 0 z}\left[\alpha_{z z z z}^{(2)} I_{\mathrm{L} z}+\left(\alpha_{z x x z}^{(2)}+\alpha_{z x z x x}^{(2)}\right) I_{\mathrm{L} x}\right]
\end{aligned}
$$

with $\Delta k_{\text {oeo }}=\omega_{\mathrm{L}}\left(2 n_{20}-n_{\mathrm{Lo}}-n_{\mathrm{Le}}\right) / c_{0}$ and

$$
\begin{array}{ll}
\alpha_{i j j i}^{(2)}=\frac{6 \omega_{\mathrm{L}}}{n_{\mathrm{Lo}} n_{\mathrm{Le}} c_{0}^{2} \varepsilon_{0}} \chi_{i j j i}^{(3) \prime \prime}\left(-\omega_{\mathrm{L}} ; \omega_{\mathrm{L}}, \omega_{\mathrm{L}},-\omega_{\mathrm{L}}\right) & i \neq j ; i=x, z ; j=z, x \\
\alpha_{i j i j}^{(2)}=\frac{6 \omega_{\mathrm{L}}}{n_{\mathrm{Lo}} n_{\mathrm{Le}} c_{0}^{2} \varepsilon_{0}} \chi_{i j i j}^{(3) \prime \prime}\left(-\omega_{\mathrm{L}} ; \omega_{\mathrm{L}}, \omega_{\mathrm{L}},-\omega_{\mathrm{L}}\right) & i \neq j ; i=x, z ; j=z, x
\end{array}
$$

In the case of negligible pump-pulse depletion by SHG Equations $23 \mathrm{~b}$ and $23 \mathrm{c}$ reduce to

$$
\begin{aligned}
& \frac{\partial}{\partial y} I_{\mathrm{L} x}=-\alpha_{\mathrm{Lo}} I_{\mathrm{L} x}-\alpha_{x x x x x}^{(2)} I_{\mathrm{L} x}^{2}-\left(\alpha_{x z z x}^{(2)}+\alpha_{x z x x z}^{(2)}\right) I_{\mathrm{L} x} I_{\mathrm{L} z} \\
& \frac{\partial}{\partial y} I_{\mathrm{L} z}=-\alpha_{\mathrm{Le}} I_{\mathrm{L} z}-\alpha_{z z z z}^{(2)} I_{\mathrm{L} z}^{2}-\left(\alpha_{z x x z}^{(2)}+\alpha_{z x z x x}^{(2)}\right) I_{\mathrm{L} x} I_{\mathrm{L} z}
\end{aligned}
$$

The second-harmonic output intensity is

$$
I_{2, \text { out }}=\left(1-R_{2 \mathrm{o}}\right) \frac{2 \omega_{2}^{2}\left|d_{15}\right|^{2} I_{\mathrm{L} x}(l) I_{\mathrm{L} z}(l)}{n_{2 \mathrm{o}} n_{\mathrm{Lo}} n_{\mathrm{Le}} c_{0}^{3} \varepsilon_{0}} \frac{1}{\left(\alpha_{2 \mathrm{o}} / 2\right)^{2}+\Delta k_{\mathrm{oeo}}^{2}}
$$

$\left(\alpha_{x z z x}^{(2)}+\alpha_{x z x z}^{(2)}\right)$ and $\left(\alpha_{z x x z}^{(2)}+\alpha_{z x z x}^{(2)}\right)$ are determined by measuring the transmission of the $x$ and $z$-components of the pump laser. $\left|d_{15}\right|$ is obtained by fitting Equations 18 and 26 to the experimental $\eta_{\mathrm{E}}^{\mathrm{SH}}$-values.

\section{Experimental}

The experimental set-up for TPA and non-phase-matched, collinear, resonant SHG (twophoton resonance at $\omega_{2}=2 \omega_{\mathrm{L}}$ ) in CdSe is shown in Fig. 1a. The picosecond pump pulses 

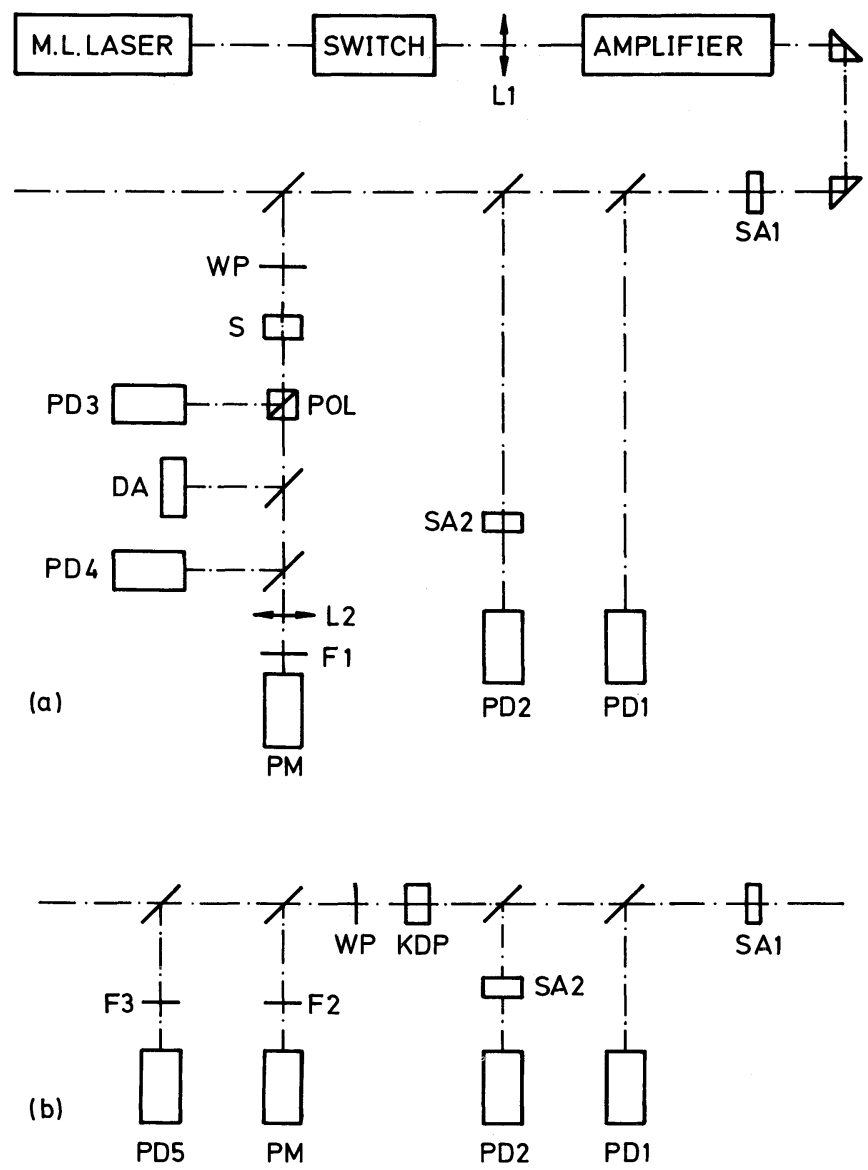

Figure 1 (a) Experimental set-up for simultaneous TPA and non-phase-matched SHG. (b) Calibration arrangement for absolute measurement of SHG energy conversion efficiency. SA1, SA2, saturable absorbers; PD1 to PD5, photodetectors; L1, L2, lenses; F1 to F3, filters; WP, half-wave plate; S, CdSe sample; DA, linear diode array; POL, Glan polarizer; PM, photomultiplier.

were generated in a passively mode-locked $\mathrm{Nd}$ : phosphate glass laser [26] (wavelength $\lambda_{\mathrm{L}}=1.054 \mu \mathrm{m}$, pulse duration $\Delta t_{\mathrm{L}} \approx 6 \mathrm{ps}$ ). Single pulses were selected with an electrooptical switch and amplified in a $\mathrm{Nd}$-phosphate glass amplifier. A saturable absorber in cell SAl was used to reduce the background energy content (dye Kodak no. 9860 in 1,2-dichloroethane, small-signal transmission $T_{0} \approx 0.04$ ). The size of the CdSe sample $\mathrm{S}$ was $12 \mathrm{~mm} \times 12 \mathrm{~mm} \times 10 \mathrm{~mm}$. The $c$-axis is parallel to a $12 \mathrm{~mm}$ edge. The peak intensity, $I_{0 \mathrm{~L}}$, of the pump pulses incident on the CdSe sample was determined by energy transmission measurement through a saturable absorber SA2 [27] (dye Kodak no. 9860 in 1,2-dichloroethane, $T_{0}=0.173$, photodetectors PD1 and PD2). The four different interaction schemes of the pump pulses with the crystal are illustrated in Fig. 2. In the case of diagonal pump pulse polarization (case 2.4) a half-wave plate WP was inserted at an angle of $22.5^{\circ}$ and the transmitted pulse components $I_{\mathrm{L} x}$ and $I_{\mathrm{L} z}$ were separated with a polarizer POL (detectors PD3 and PD4). The beam profile of the transmitted pump pulses was monitored with a 
(i) $k_{L} \| c$ :

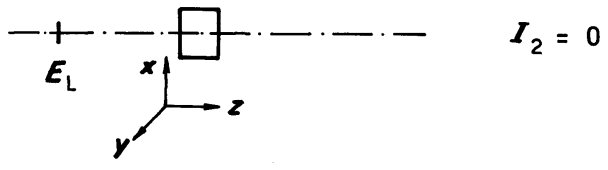

(ii) $k_{L} \perp c, E_{L} \perp c: 00-e$

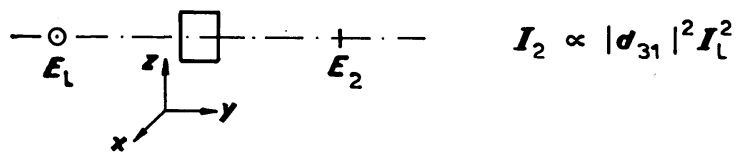

(iii) $k_{L} \perp c, E_{L} \| c:$ : ee $-\mathrm{e}$

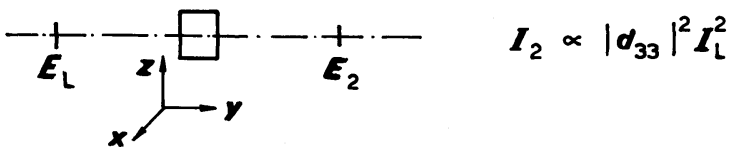

(iv) $\boldsymbol{k} \perp \boldsymbol{c}, \Varangle(\boldsymbol{E}, \boldsymbol{c})=45^{\circ}:$ oe $\rightarrow 0$

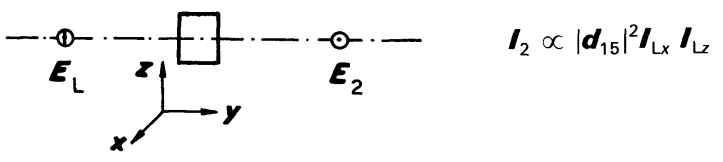

Figure 2 Interaction schemes.

linear photodiode array. The generated second-harmonic light was detected with a photomultiplier PM (S20 spectral characteristic).

For absolute measurements of the second-harmonic energy conversion efficiency, $\eta_{\mathrm{E}}^{\mathrm{SH}}$, the photomultiplier sensitivity was calibrated by measuring the second-harmonic signal under high conversion efficiency conditions caused by phase-matched SHG in a KDP-crystal (Fig. 1b). In this case $\eta_{\mathrm{E}}^{\mathrm{SH}}$ was determined by the reduction of the pump pulse signal (photodetectors PD5 and PD1).

The refractive indices $n_{\mathrm{Lo}}$ and $n_{\mathrm{Le}}$ of CdSe were taken from the literature [3, 28-31] (see Table III). The linear absorption coefficients $\alpha_{\mathrm{Lo}}$ and $\alpha_{\mathrm{Le}}$ were calculated from the transmission measurements at low pump pulse intensities (Figs 3 to 5 ) applying the formula $[32,33]$

with

$$
T_{\mathrm{E}, i}=\frac{\left(1-R_{\mathrm{L}, i}\right) \exp \left(-\alpha_{\mathrm{L}, i} l\right)}{1-R_{\mathrm{L}, i}^{2} \exp \left(-2 \alpha_{\mathrm{L}, i} l\right)}
$$

$$
R_{\mathrm{L}, i}=\frac{\left(n_{\mathrm{L}, i}-1\right)^{2}+\kappa_{\mathrm{L}, i}^{2}}{\left(n_{\mathrm{L}, i}+1\right)^{2}+\kappa_{\mathrm{L}, i}^{2}}
$$




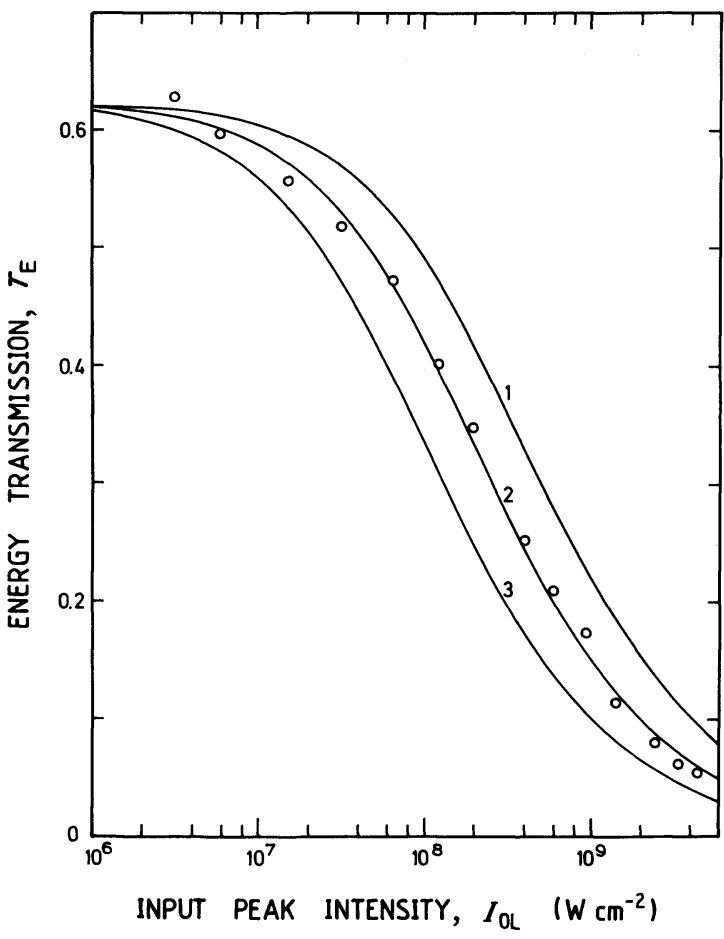

Figure 3 Energy transmission for pulse propagation parallel to the optical axis (case 2.1). Sample length $I=12 \mathrm{~mm}$. Curves are calculated (Equations 12 and 13) for $\alpha_{x x x x}^{(2)}=$ $9 \times 10^{-9} \mathrm{~cm} \mathrm{~W}^{-1}(1), \quad 1.8 \times 10^{-8} \mathrm{~cm} \mathrm{~W}^{-1}$ (2) and $3.6 \times 10^{-8} \mathrm{~cm} \mathrm{~W}^{-1}(3)$.

TABLE III Parameters of CdSe. $\lambda_{\mathrm{L}}=1.054 \mu \mathrm{m}, \lambda_{2}=527 \mathrm{~nm}$, room temperature

\begin{tabular}{lll}
\hline Parameter & Value & Reference \\
\hline$n_{\mathrm{Lo}}$ & 2.537 & 28 \\
$n_{\mathrm{Le}}$ & 2.5585 & 28 \\
$n_{2 \mathrm{o}}$ & 2.658 & 34 \\
$n_{2 \mathrm{e}}$ & 2.666 & 34 \\
$\alpha_{\mathrm{Lo}}$ & $(0.075 \pm 0.05) \mathrm{cm}^{-1}$ & This work, Equation 27 \\
$\alpha_{\mathrm{Le}}$ & $(0.10 \pm 0.05) \mathrm{cm}^{-1}$ & This work, Equation 27 \\
$\kappa_{2 \mathrm{o}}$ & $0.50 \pm 0.04$ & 34 \\
$\kappa_{2 \mathrm{e}}$ & $0.624 \pm 0.04$ & 34 \\
$\alpha_{2 \mathrm{o}}$ & $(1.19 \pm 0.10) \times 10^{5} \mathrm{~cm}^{-1}$ & Equation 29 \\
$\alpha_{2 \mathrm{e}}$ & $(1.49 \pm 0.10) \times 10^{5} \mathrm{~cm}^{-1}$ & Equation 29 \\
$\Delta k_{\mathrm{ooe}}$ & $1.54 \times 10^{4} \mathrm{~cm}^{-1}$ & Equation 4 \\
$\Delta k_{\text {eee }}$ & $1.28 \times 10^{4} \mathrm{~cm}^{-1}$ & Equation 4 \\
$\Delta k_{\mathrm{oeo}}$ & $1.31 \times 10^{4} \mathrm{~cm}^{-1}$ & Equation 4 \\
\hline
\end{tabular}

and

$$
\kappa_{\mathrm{L}, i}=\frac{\alpha_{\mathrm{L}, i} c_{0}}{2 \omega_{\mathrm{L}}}
$$

where $i$ denotes o or e.

The refractive indices $n_{20}$ and $n_{2 \mathrm{e}}$ and the extinction coefficients $\kappa_{20}$ and $\kappa_{2 \mathrm{e}}$ were determined by reflection measurements $[34,35]$ and are listed in Table III. The optical constants of CdSe over a wide wavelength region are reported in [25]. 

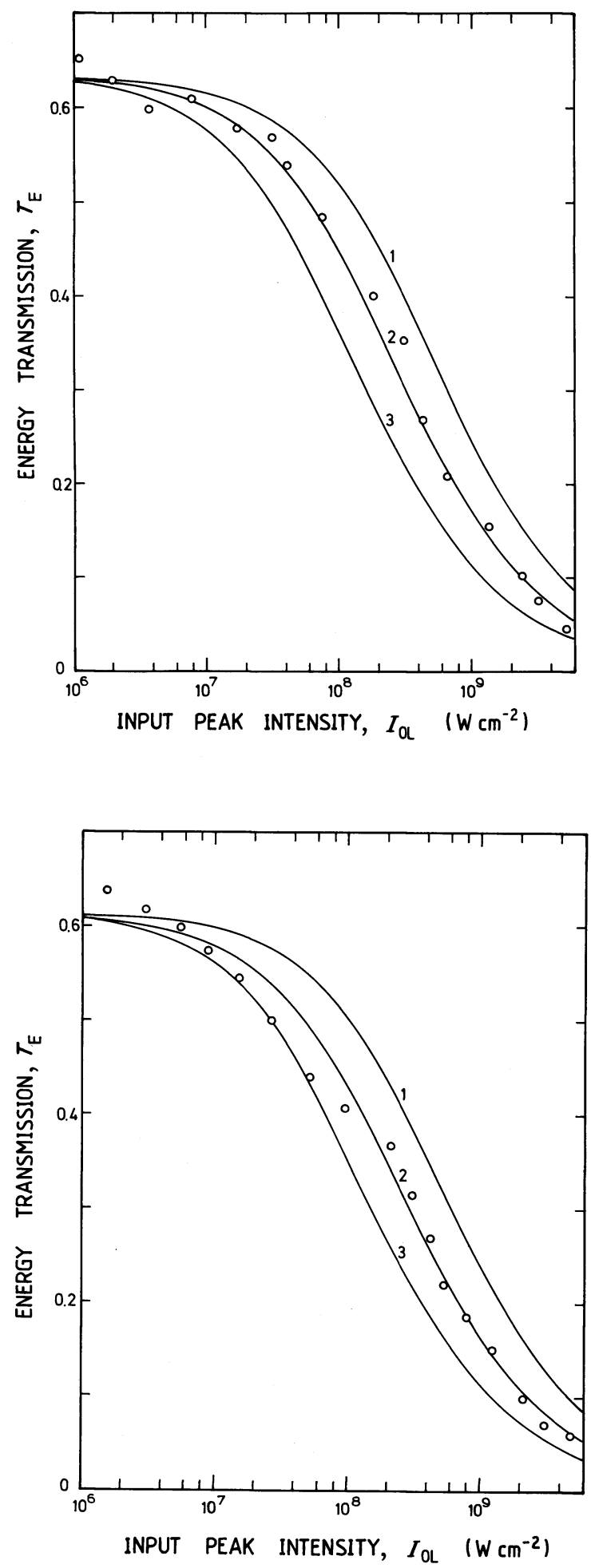

Figure 4 Energy transmission for pulse propagation and pulse polarization perpendicular to the optical axis (case 2.2). Sample length $I=1 \mathrm{~cm}$. Curves are calculated (Equations 12 and 13) for $\alpha_{x x x x}^{(2)}=9 \times 10^{-9} \mathrm{~cm} \mathrm{~W}^{-1}(1), 1.8 \times$ $10^{-8} \mathrm{~cm} \mathrm{~W}^{-1}(2)$ and $3.6 \times 10^{-8} \mathrm{~cm} \mathrm{~W}^{-1}$ (3).

Figure 5 Energy transmission for pulse propagation perpendicular to optical axis and pulse polarization parallel to the optical axis (case 2.3). Sample length $/=1 \mathrm{~cm}$. Curves are calculated (Equations 12 and 13) for $\alpha_{z z z z}^{(2)}=9 \times 10^{-9} \mathrm{~cm} \mathrm{~W}^{-1}$ (1), $1.8 \times$ $10^{-8} \mathrm{~cm} \mathrm{~W}^{-1}$ (2) and $3.6 \times 10^{-8} \mathrm{~cm} \mathrm{~W}^{-1}$ (3). 


\section{Results}

The TPA results are shown in Figs 3 to 6 . The energy transmissions for $\boldsymbol{k}_{\mathrm{L}} \| \boldsymbol{c}$ (case 2.1) are shown in Fig. 3. For this crystal orientation no SHG occurs. The circles are experimental points and the curves are calculated (Equations 12 and 13). The best-fitting TPA coefficient is $\alpha_{r x x x}^{(2)}=1.8 \times 10^{-8} \mathrm{~cm} \mathrm{~W}^{-1}$ (see Table II). Curve 2 fits the experimental points over the whole intensity region investigated. This fact indicates that excited-state absorption of two-photon generated carriers plays a negligible role in the interaction of the picosecond pump pulses with the crystal (excited-state absorption not included in the theory).

The pump-pulse energy transmission for $\boldsymbol{k}_{\mathrm{L}} \perp \boldsymbol{c}$ and $\boldsymbol{E}_{\mathrm{L}} \perp \boldsymbol{c}$ (case 2.2, oo $\rightarrow$ e secondharmonic interaction) is shown in Fig. 4. The experimental data points are best fitted with the same value of the TPA coefficient $\alpha_{x x x x}^{(2)}$ as in case 2.1 , indicating that the oo $\rightarrow$ e SHG has no measurable influence on the energy transmission. In the numerical simulations (solution of Equations 14a and b) below it will be seen that the second-harmonic signal generated (Fig. 7) is two orders of magnitude too small for a measurable pump-pulse reduction by SHG $\left(\left|d_{31}\right|\right.$ is one order of magnitude too small).

The pump-pulse energy transmission for $\boldsymbol{k}_{\mathrm{L}} \perp \boldsymbol{c}$ and $\boldsymbol{E}_{\mathrm{L}} \| \boldsymbol{c}$ (case 2.3, ee $\rightarrow$ e secondharmonic interaction) is shown in Fig. 5. The best-fitting TPA coefficient is $\alpha_{z z z z}^{(2)}=1.8 \times$ $10^{-8} \mathrm{~cm} \mathrm{~W}^{-1}$ (see Table II). Again, the SHG (ee $\rightarrow$ e interaction, Fig. 7) causes no measurable pump-pulse reduction.

For $\boldsymbol{k}_{\mathrm{L}} \perp \boldsymbol{c}$ and $\measuredangle\left(E_{\mathrm{L}}, \boldsymbol{c}\right)=45^{\circ}$ (case 2.4 , oe $\rightarrow$ o second-harmonic interaction) the TPA results are shown in Fig. 6. The energy transmission $T_{\mathrm{E}, x}$ and $T_{\mathrm{E}, z}$ are plotted against the input pump-pulse peak intensity $I_{0 \mathrm{~L}, \text { in }}$ (Equations $25 \mathrm{a}$ and $\mathrm{b}$ ). The experimental points are best fitted for $\alpha_{x z z x}^{(2)}+\alpha_{x z x z}^{(2)}=3 \times 10^{-9} \mathrm{~cm} \mathrm{~W}^{-1}$ and $\alpha_{z x x z}^{(2)}+\alpha_{z x z x}^{(2)}=3 \times 10^{-9} \mathrm{~cm} \mathrm{~W}^{-1}$

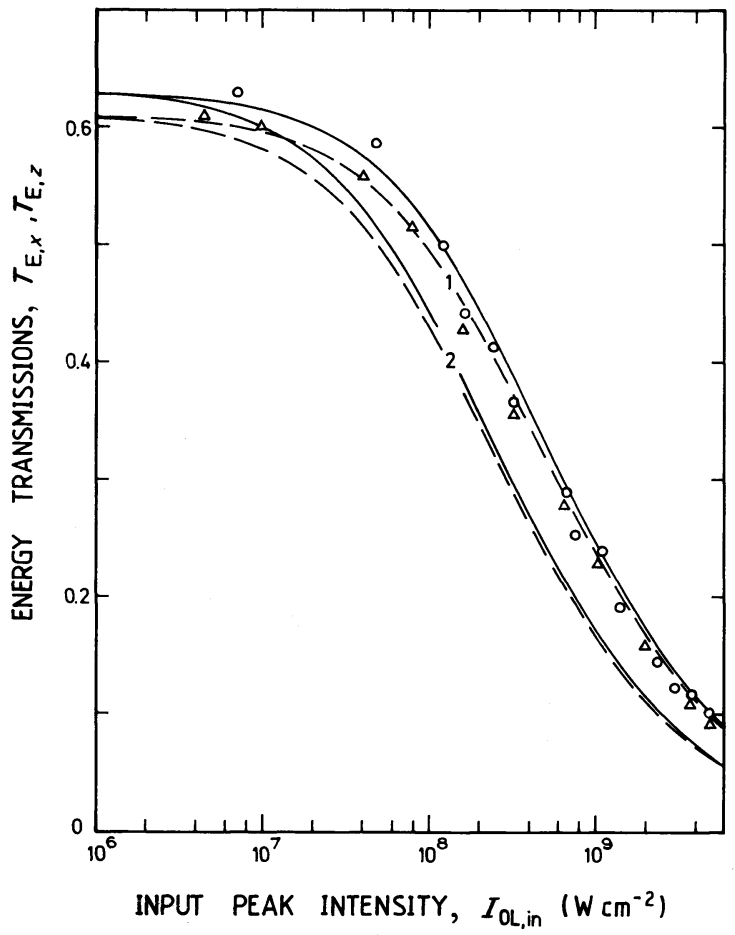

Figure 6 Energy transmission for pulse propagation perpendicular to the optical axis and pulse polarization diagonal to the optical axis (case 2.4). Sample length $/=$ $1 \mathrm{~cm}$. (O-O) Ordinary polarized components $\left(T_{E, x}\right),(\Delta---\Delta)$ extraordinary polarized component $\left(T_{E, z}\right)$. Curves 1, $\alpha_{x x x x}^{(2)}=$ $\alpha_{z z z z}^{(2)}=1.8 \times 10^{-8} \mathrm{~cm} \mathrm{~W}^{-1}$ and $\alpha_{x z z x}^{(2)}+\alpha_{x z x z}^{(2)}=$ $\alpha_{z x x z}^{(2)}+\alpha_{z x z x}^{(2)}=0$; curves 2, $\alpha_{x x x x}^{(2)}=\alpha_{z z z z}^{(2)}=\alpha_{x z z x}^{(2)}+$ $\alpha_{x z x z}^{(2)}=\alpha_{z x x z}^{(2)}+\alpha_{z x z x}^{(2)}=1.8 \times 10^{-8} \mathrm{~cm} \mathrm{~W}^{-1}$. 


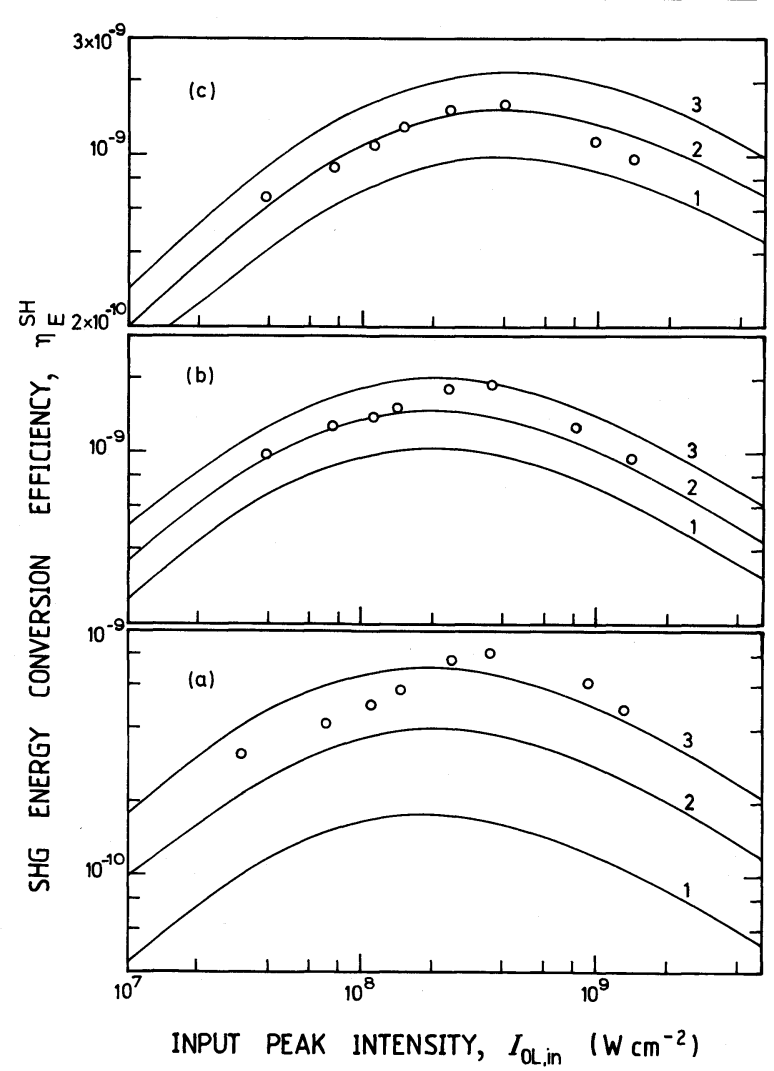

Figure 7 Energy conversion efficiency of second-harmonic light. (a) oo $\rightarrow$ e Interaction (case 2.2). Curves are for $\alpha_{x x x x}^{(2)}=$ $1.8 \times 10^{-8} \mathrm{~cm} \mathrm{~W}^{-1}$ and $\left|d_{31}\right|=1.23 \times$ $10^{-11} \mathrm{~m} \mathrm{~V}^{-1}$ (1), $1.84 \times 10^{-11} \mathrm{mV}^{-1}$ (2), $2.46 \times 10^{-11} \mathrm{mV}^{-1}$ (3). (b) ee $\rightarrow$ e Interaction (case 2.3). Curves are calculated for $\alpha_{z z z z}^{(2)}=1.8 \times 10^{-8} \mathrm{~cm} \mathrm{~W}^{-1}$ and $\left|d_{33}\right|=3.1 \times$ $10^{-11} \mathrm{~m} \mathrm{~V}^{-1}(1), 3.7 \times 10^{-11} \mathrm{~m} \mathrm{~V}^{-1}(2), 4.3$ $\times 10^{-11} \mathrm{mV}^{-1}$ (3). (c) oe $\rightarrow$ o Interation (case 2.4). Curves are for $\alpha_{x x x x}^{(2)}=\alpha_{z 222}^{(2)}=$ $1.8 \times 10^{-8} \mathrm{~cm} \mathrm{~W}^{-1}, \alpha_{x z z x}^{(2)}+\alpha_{x z x z}^{(2)}=\alpha_{z x x z}^{(2)}+\alpha_{z x z x}^{(2)}=$ $3 \times 10^{-9} \mathrm{~cm} \mathrm{~W}^{-1}$ and (1) $\left|d_{15}\right|=1.8 \times$ $10^{-11} \mathrm{~m} \mathrm{~V}^{-1}$, (2) $\left|d_{15}\right|=2.26 \times 10^{-11} \mathrm{~m} \mathrm{~V}^{-1}$ and (3) $\left|d_{15}\right|=2.71 \times 10^{-11} \mathrm{~m} \mathrm{~V}^{-1}$.

(Table II). The second-harmonic light generated (Fig. 7) is too weak to contribute measurably to the pump-pulse absorption.

The SHG energy conversion efficiencies $\eta_{\mathrm{E}}^{\mathrm{SH}}$ are plotted against the pump-pulse input peak intensity $I_{0 \mathrm{~L} \text {,in }}$ in Fig. 7. The curves were calculated for the situation of no pump-pulse reduction by SHG (Fig. 7a, Equations 17 and 18 for oo $\rightarrow$ e interaction; Fig. 7b, Equations 22 and 18 for ee $\rightarrow$ e interaction; and Fig. 7c, Equations 26 and 18 for oe $\rightarrow$ o interaction). The best-fitting second-harmonic susceptibilities are listed in Table I. The $d$-values obtained are approximately $20 \%$ smaller than the corresponding $d$-values at $\lambda_{\mathrm{L}}=10.6 \mu \mathrm{m}$.

\section{Simulations}

The influence of the resonant SHG (strong linear absorption of generated second-harmonic light) on the pump-pulse transmission was studied by solving Equations 14a and b numerically (oo $\rightarrow$ e interaction). The CdSe parameters of Table III were applied except where noted otherwise. Only intensity transmissions, $T_{1}$, and second-harmonic intensity conversion efficiencies, $\eta_{1}^{\mathrm{SH}}$, are presented (spatial and temporal rectangular pulse shape).

\subsection{Second-harmonic generation without TPA}

The full curves in Fig. 8 show the pump-pulse transmission in the case of non-phasematched resonant SHG $\left(\alpha_{x x x x}^{(2)}=0\right)$. $\left|d_{31}\right|$ was varied. The same results are obtained for $d_{31}$ real or imaginary. For comparison pump-pulse transmissions due to TPA are included (broken 


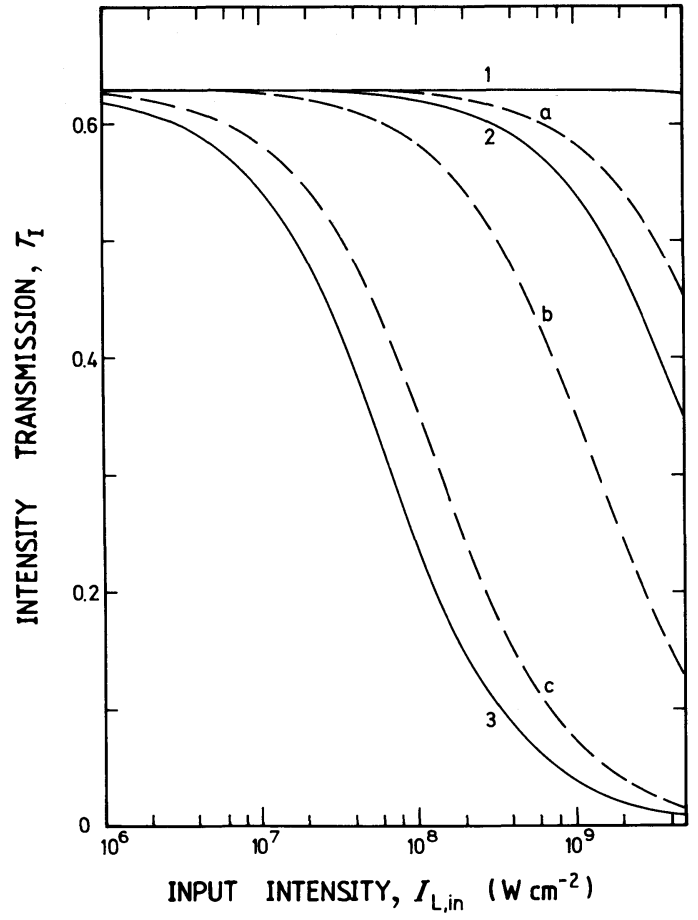

Figure 8 Influence of SHG on pump-pulse transmission. Sample length $I=1 \mathrm{~cm} . \boldsymbol{k}_{\mathrm{L}} \perp \boldsymbol{c}$ and $\boldsymbol{E}_{\mathrm{L}} \perp \boldsymbol{c}$. $(-)$ Only SHG $\left(\alpha_{x x x x}^{(2)}=0\right)$. 1, $\left|d_{31}\right|=10^{-11} \mathrm{~m} \mathrm{~V}^{-1} ; 2,\left|d_{31}\right|=10^{-10} \mathrm{~m} \mathrm{~V}^{-1} ; 3$, $\left|d_{31}\right|=10^{-9} \mathrm{~m} \mathrm{~V}^{-1}$. (-- ) Only TPA $\left(d_{31}=0\right)$. a, $\alpha_{x x x x}^{(2)}=10^{-10} \mathrm{~cm} \mathrm{~W}^{-1} ; b, \alpha_{x x x x}^{(2)}=10^{-9} \mathrm{~cm} \mathrm{~W}^{-1}$; c, $\alpha_{x x x x}^{(2)}=10^{-8} \mathrm{~cm} \mathrm{~W}^{-1}$.

curves, $\left.d_{31}=0\right)$. The same functional dependences are found for $T_{\mathrm{I}}\left(I_{\mathrm{L}, \text { in }}\right)$ in the case of SHG and TPA.

The curves in Fig. 9 show the second-harmonic efficiencies $\eta_{1}^{\mathrm{SH}}$ plotted against the pump laser intensity $I_{\mathrm{L}, \text { in }}$. At low pump intensities $\eta_{\mathrm{I}}^{\mathrm{SH}}$ rises proportionally to $I_{\mathrm{L}, \text { in }}$. Then the second-harmonic signal becomes strong enough to reduce the pump-pulse transmission and $\eta_{1}^{\mathrm{SH}}$ approaches a maximum value. At even higher pump-pulse intensities the output pump intensity approaches a constant value (see Equation 11, power-limiting action [17]) generating a constant second-harmonic signal, and consequently $\eta_{1}^{\mathrm{SH}}=I_{2, \text { out }} / I_{\mathrm{L}, \text { in }}$ decreases proportionally to $1 / I_{\mathrm{L}, \text { in }}$.

The situation of phase-matched resonant $\operatorname{SHG}\left(\Delta k_{\mathrm{ooe}}=0, \alpha_{x x x x}^{(2)}=0\right)$ has been analysed. The intensity transmissions are slightly higher and the second-harmonic conversion efficiencies are slightly lower than in the case of non-phase-matched resonant SHG. However, the differences are so small that the curves coincide within the line strength. For CdSe (data of Table III) the linear absorption at the second-harmonic frequency dominates over the phase-mismatch (see Equation 16).

The maximum second-harmonic conversion efficiency $\eta_{1, \max }^{\mathrm{SH}}$ may be estimated by considering that $I_{2}$ approaches an upper limit after an effective interaction length of $l_{\text {eff }}=\alpha_{2}^{-1}$, and that the generated second-harmonic light is absorbed $l / l_{\mathrm{eff}}$ times in the crystal. For $\eta_{\mathrm{I}, \text { max }}^{\mathrm{SH}} l \alpha_{2}=\left(1-R_{\mathrm{L}}\right)\left(1-R_{2}\right)$ the complete input light is converted to second-harmonic light which is absorbed. A maximum second-harmonic efficiency of

$$
\eta_{1, \text { max }}^{\mathrm{SH}} \approx \frac{\left(1-R_{\mathrm{L}}\right)\left(1-R_{2}\right)}{l \alpha_{2}}
$$

is estimated by these considerations. 


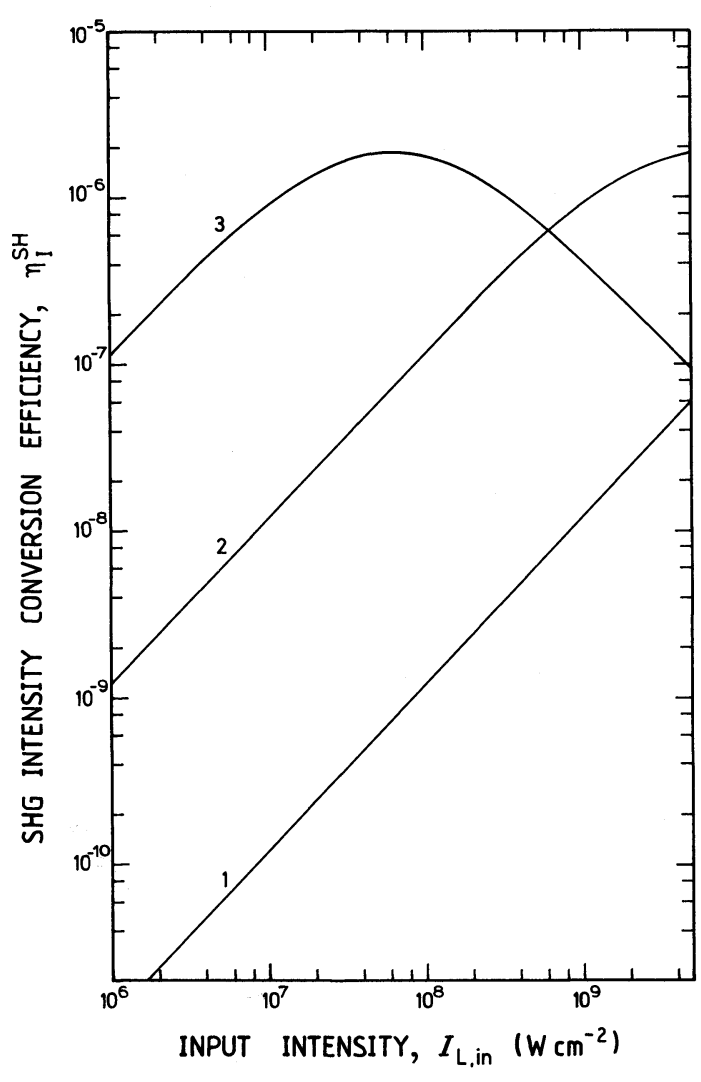

Figure 9 Second-harmonic generation without TPA $\left(\alpha_{x x x x}^{(2)}=0\right)$. Sample length $/=1 \mathrm{~cm}, \boldsymbol{k}_{\mathrm{L}} \perp \boldsymbol{c}$ and $\boldsymbol{E}_{\mathrm{L}} \perp$ c. $1,\left|d_{31}\right|=10^{-11} \mathrm{~m} \mathrm{~V}^{-1} ; 2,\left|d_{31}\right|=$ $10^{-10} \mathrm{~m} \mathrm{~V}^{-1} ; 3,\left|d_{31}\right|=10^{-9} \mathrm{~m} \mathrm{~V}^{-1}$.

\subsection{Simultaneous SHG and TPA}

The TPA coefficient is set to the experimental value of $\alpha_{x x x x}^{(2)}=1.8 \times 10^{-8} \mathrm{~cm} \mathrm{~W}^{-1}$ and $\left|d_{31}\right|$ is varied. The intensity transmissions $T_{1}\left(I_{\mathrm{L}, \text { in }}\right)$ and the second-harmonic efficiencies $\eta_{1}^{\mathrm{SH}}\left(I_{\mathrm{L} \text {.in }}\right)$ are shown in Figs 10 and 11, respectively. Up to $\left|d_{31}\right| \approx 2 \times 10^{-10} \mathrm{~m} \mathrm{~V}^{-1}$ the SHG does not contribute an observable amount to the intensity transmission. The experimental $\left|d_{31}\right|$-value (Fig. 7 and Table I) is an order of magnitude lower. Therefore, the SHG in CdSe does not influence the TPA.

The second-harmonic conversion efficiency $\eta_{1}^{\mathrm{SH}}$ is lowered by the TPA since the pump intensity $I_{\mathrm{L}}(l)$ at the exit surface of the crystal is responsible for the output secondharmonic signal. This fact is clearly seen by comparing the dotted curve $\left(\alpha_{x x x x}^{(2)}=0\right.$, $\left.\left|d_{31}\right|=10^{-9} \mathrm{~m} \mathrm{~V}^{-1}\right)$ and the full curve $3\left(\alpha_{x x x x}^{(2)}=1.8 \times 10^{-8} \mathrm{~cm} \mathrm{~W}^{-1},\left|d_{31}\right|=10^{-9} \mathrm{~m} \mathrm{~V}^{-1}\right)$ of Fig. 11.

\section{Conclusions}

The simultaneous occurrence of TPA and resonant non-phase-matched SHG in CdSe has been studied experimentally and theoretically. The complete second-harmonic susceptibility tensor and some components of the TPA coefficient tensor have been determined. The second-harmonic susceptibility components at $\lambda_{\mathrm{L}}=1.054 \mu \mathrm{m}$ are approximately $20 \%$ smaller than the corresponding components at $\lambda_{\mathrm{L}}=10.6 \mu \mathrm{m}$. The TPA in CdSe is not 

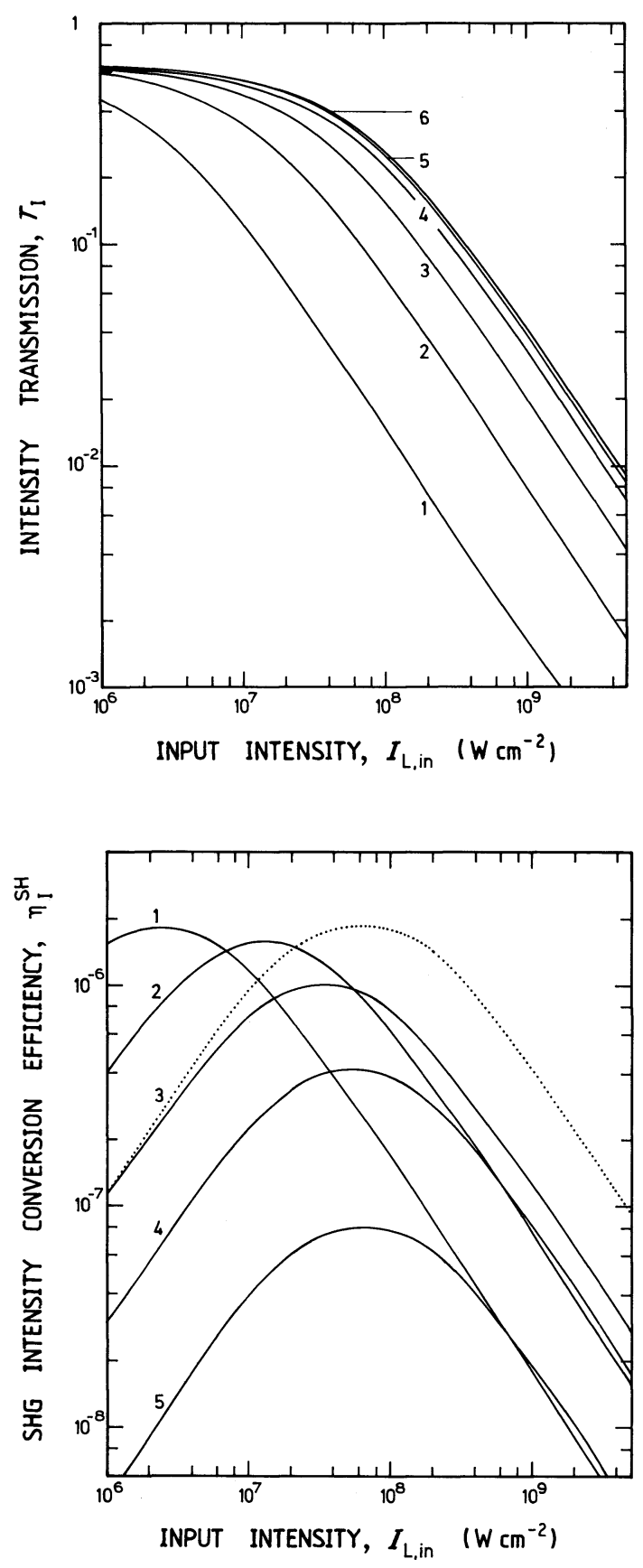

Figure 10 Influence of combined TPA and SHG on pump-pulse transmission. Sample length $I=1 \mathrm{~cm} . \quad \boldsymbol{k}_{\mathrm{L}} \perp \boldsymbol{C}$ and $\boldsymbol{E}_{\mathrm{L}} \perp \boldsymbol{c} . \alpha_{x x x x}^{(2)}=1.8 \times$ $10^{-8} \mathrm{~cm} \mathrm{~W}^{-1}$. The $\left|d_{31}\right|$-values are (1) $5 \times 10^{-9}$, (2) $2 \times 10^{-9}$, (3) $10^{-9}$, (4) $5 \times 10^{-10}$, (5) $2 \times 10^{-10}$ and (6) $0 \mathrm{mV}^{-1}$.

Figure 11 Influence of TPA on SHG. The full curves are for the same parameters as the curves in Fig. 10. The dotted curve is for $\alpha_{x x x x}^{(2)}=0$ and $\left|d_{31}\right|=10^{-9} \mathrm{~m} \mathrm{~V}^{-1}$.

influenced by the SHG occurring simultaneously because the second-order susceptibility components are an order of magnitude too small.

The measured energy transmissions in CdSe plotted against the pump-pulse peak intensity (Figs 3 to 6) may be used as calibration curves for the peak intensity detection of picosecond light pulses by energy transmission measurement [36, 37]. 


\section{References}

1. D'ANS LAX, 'Taschenbuch für Chemiker and Physiker', Vol. 1, edited by E. Lax (Springer-Verlag, Berlin, 1967).

2. C. K. N. PATEL, Phys. Rev. Lett. 16 (1966) 613.

3. R. L. HERBST and R. L. BYER, Appl. Phys. Lett. 19 (1971) 527.

4. M. M. CHOY and R. L. BYER, Phys. Rev. B14 (1976) 1693.

5. R. A. SOREFF and H. W. MOOS, J. Appl. Phys. 35 (1964) 2152.

6. N. G. BASOV, A. Z. GRASIUK, V. F. EFINKOV, I. G. ZUBAREV, V. A. KATUliN and J. M. POPOV, J. Phys. Soc. Jpn Suppl. 21 (1966) 276.

7. A. Z. GRASYUK, I. G. ZUBAREV and A. N. MENTSER, Soviet Phys. - Solid St. 10 (1968) 427.

8. J. M. RALSTON and R. K. CHANG, Appl. Phys. Lett. 15 (1969) 164.

9. Idem, Optoelectronics 1 (1969) 182.

10. F. BRYUKNER, V. S. DNEPROVSKII and V. U. KHATTATOV, Soviet J. Quantum Electron. 4 (1974) 749.

11. V. S. DNEPROVSKII and SH. M. OK, ibid. 6 (1976) 298.

12. D. C. HANNA and A. J. TURNER, Opt. Quantum Electron. 8 (1976) 213.

13. J. H. BECHTEL and W. L. SMITH, Phys. Rev. B13 (1976) 3515.

14. M. BASS, E. W. VanSTRYLAND and A. F. STEWARD, Appl. Phys. Lett. 34 (1979) 142.

15. A. F. STEWARD and M. BASS, ibid. 37 (1980) 1040.

16. E. W. VanSTRYLAND and M. A. WOODALL, NBS Spec. Publ. 620 (1980) 50.

17. E. W. VanSTRYLAND, H. VANHERZEELE, M. J. SOILEAU, A. L. SMIRL, S. GUHA and T. F. BOGGERS, Opt. Engng 24 (1985) 613.

18. W. L. SMITH, 'CRC Handbook of Laser Science and Technology', Vol. III: 'Optical Materials: Part 1', edited by M. J. Weber (CRC Press, Boca Raton, Florida, 1986).

19. Y. R. SHEN, 'The Principles of Nonlinear Optics' (Wiley, New York, 1984).

20. M. SCHUBERT and B. W. WILHELMI, 'Nonlinear Optics and Quantum Electronics' (Wiley, New York, 1986).

21. A. PENZKOFER and W. LEUPACHER, Opt. Quantum Electron. 20 (1988) 227.

22. F. ZERNIKE and J. E. MIDWINTER, 'Applied Nonlinear Optics' (Wiley, New York, 1973).

23. M. THALHAMMER and A. PENZKOFER, Appl. Phys. B32 (1983) 137.

24. P. N. BUTCHER, 'Nonlinear Optical Phenomena' (Bulletin 200, Engineering Experiment Station, Ohio State University, Columbus, Ohio, 1965).

25. M. CARDona and G. HARBeKe, Phys. Rev. A137 (1965) 1467.

26. A. PENZKOFER and F. GRAF, Opt. Quantum Electron. 17 (1985) 219.

27. A. PENZKOFER, D. VON DER LINDE and A. LAUBEREAU, Opt. Commun. 4 (1972) 377.

28. M. P. LiSitSA, L. F. GUDYMENKO, V. N. MALINKO and S. F. TEREKhoVA, Phys. Status Solidi 31 (1969) 389.

29. S. A. GEIDUR and A. D. YASKOV, Opt. Spectrosc. 48 (1980) 618.

30. A. N. PIKHTIN and A. D. YAS'KOV, Soviet Phys. Semiconductors 15 (1981) 8.

31. B. JENSEN and A. TORABI, J. Opt. Soc. Am. B3 (1986) 857.

32. J. I. PANKOVE, 'Optical Processes in Semiconductors' (Dover, New York, 1971).

33. A. PENZKOFER and A. A. BUGAYEV, Opt. Quantum Electron. 21 (1989) 283.

34. X. BAO, M. SCHÄFFNER and A. PENZKOFER, to be published.

35. Y. LU and A. PENZKOFER, Appl. Opt. 25 (1986) 221.

36. A. PENZKOFER and W. FALKENSTEIN, Opt. Commun. 17 (1976) 1.

37. W. BLAU and A. PENZKOFER, ibid. 36 (1981) 419.

38. R. W. MINCK, R. W. TERHUNE and C. C. WANG, Appl. Opt. 5 (1966) 1595. 